\title{
MODEL DEVELOPMENT OF PHYSICAL EXERCISE GOALKEEPER OF FOOTBALL WITH CIRCUIT
}

\author{
Rizki Apriliyanto,* Mulyana,** James Tangkudung,*** \\ Universitas Muhammadiyah Jember \\ Universitas Negeri Jakarta \\ rizkiapriliyanto@unmuhjember.ac.id \\ Mulyana@unj.ac.id \\ James.tangkudung@unj.ac.id
}

\begin{abstract}
This study aims to develop models of physical exercise soccer goalie by using the circuit method. Research development is packaged in the form of circuit training models to improve the physical condition of football goalkeeper. Methods used are research and development (Research and Development) of the Borg \& Gall with 10 steps. Feasibility models and evaluated by five expert sports. Those are 2 experts of coaching and 3 experts of goalkeeper. While the subject of research is the goalkeeper in Banyuwangi. This research begins with needs analysis, planning, product, testing, revision and final products. The results show that this model is feasible to use the product goalkeeper. In the test results for small groups and large groups can be explained that the model as a whole can do well. In a test of the effectiveness of the mean value for the group amounted to 42 221 circuit and the value of the conventional group amounted to 33167 -count value 3,447 such results can be declared effective circuit training models to improve the physical abilities keeper.It shows that the model of physical exercise with circuit can be used by the goalkeeper in football and can effectively improve the physical abilities soccer goalkeeper.
\end{abstract}

Keywords: Exercise Models, Methods Circuits, Physical Condition.

Let us consider the following news excerpts:

“...Sixteen rescues are performed several times in very close distance. German goalkeeper, Manuel Neuer, several times ran out of the penalty box to block the ball strikes the Algerian players. Similar praise addressed to Ochoa, the goalkeeper Mexican who managed to draw against the Brazilian team 0-0 in a match of Group A. The goalkeeper of Costa Rica, Keylor Navas, also is a hero for his team when playing with 10 players. They beat Greece in the round of $16 . "$

"News snippets above are events that happen at the World Cup 2014, rescue carried out by the third goalkeeper illustrates that the success of a football team 
cannot be separated from the role of the keeper. Its presence is needed in anticipation of an attack of the opponent. Rescue is often done could usher in which he defended the team's victory. All this is evidence that the contribution of the goalkeeper have contributed to the success of a football team.

Absolutely, to be a reliable goalkeeper as the news snippets above it takes quite a long process, all acquired cannot be done instantly. Development of continuous a and systematic workout is something that must considered. Without such a process, then certainly a failure to foster football goalkeeper is only a matter of time. The reflection gives a warning for a coach in skill development efforts of goalkeeper.

Recognizing and understanding above, it should be fostering a goalkeeper in soccer game must begin at a young age. It is important, because a young age is an initial phase in achieving peak performance. When in a young age is not handled well, do not expect the coaching which has run can produce a competent goalkeeper. Therefore, it is no exaggeration if in a young age is also referred to as the foundation to move on to higher level. Like a building, when the foundation is fragile then surely the standing building will not be able to stand firmly. The same thing is true in soccer coaching. When a young age is controlled well, then the ultimate achievement will be more easily achieved.

Not infrequently, the success in coaching goalkeeper soccer is determined from how the administration portion and training methods are used. This is important considering the portion and training methods must be tailored to the characteristics and needs of the goalkeeper, if it is not, so it is feared the development which has taken place will not run optimally. It could be because it does not reflect the characteristics of the goalkeeper; the training process does not have a significant effect or the imposition of exercises that do not fit the needs of soccer goalkeeper could adversely affect the growth and psychological development which in turn will lead to the meaninglessness in the training process.

Although many factors influence to achieve peak performance, a factor of the training plan should be a priority. Fail in achievement means failure in planning the exercise, therefore, in order to develop the ability of a goalkeeper needs to be designed exercises that are tailored to the characteristics and needs of keeper. The designing an exercise would not be easy and will not be necessarily controled for granted, it is necessary to do in-depth theoretical studies. The role of the coach in this case becomes very crucial, because knowledge of the coaching methodology concepts become an integral part in delivering soccer goalie to achieve peak performance. It could also be said what is gained by the keeper is a mirror of what is given from the coach. Therefore planning exercise program designed by a trainer depends on how deep knowledge of the concept of sports coaching methodology.

From the explanation above, the exercise pattern should be oriented to the characteristics and needs of the keeper. Knowledge of the characteristics of the goalkeeper is the key in choosing the materials and methods of practice what is 
suitable to be applied. This is called exercise to be adapted to the needs of the keeper. Therefore, the role of the coach becomes the essential in determining the exercise. It is a smart move if the training process based on theoritical studies. That is to promote the concept of methodology in providing the stimulus of exercise is a necessity.

With experience as a soccer goalkeeper, the author moved to determine the real field conditions. Has this exercise undertaken during exercise methodology embracing the concept?

Has the exercise been adapted to the characteristics and needs of the goalkeeper? This is important, because if the exercise is not adapted to the characteristics and concepts soccer goalkeeper existing methodology, the failure to develop a quality goalkeeper is only a matter of time and intensity.

To overcome curiosity, the author moved to do initial research on a soccer school and local clubs in Banyuwangi. From the observations are obtained information that the practice of physical exercises that have been undertaken by the keeper using drill. Other findings, practice is applied only rely on intuition. What to remember when it was what trained on the spot. In addition at the same time also conducted interviews with the coach, said that for this lack in physical training manuals for the goalkeeper into the limitations in providing a menu of physical exercise keeper.

For the reason, It needs innovation in the procession physical exercise keeper. In addition to observation, the support data also is needed to sharpen the problems in the field. Support data is intended to reveal the results of a needs analysis. Acquisition of a needs analysis by distributing a questionnaire to the athletes goalkeeper, from the distribution of the questionnaire can be concluded that the development of models of physical exercise for the goalkeeper agree to be developed with a percentage of $64.96 \%$ (sixty four point ninety-six percent). With the supporting data reinforced the practice of physical models that need to be developed.

It can be concluded that the need for the presence of physical exercise models goalkeeper is urgent. That is why it needs to there be real action about the model of physical exercise keeper. Wise way in addressing the above problems is necessary for an investigation. The study is meant to develop a model of exercise physical football goalkeeper. From the findings of this analysis, the authors perform theoretical studies to determine the choice of training methods in the use of what might be used assessment which can be done by looking at the results.

The results of the Minakshi Pathak (2015) reported that, group training method further maximize circuit in terms of capacity Cardiovascular badminton players. The results of research from N. Akilan and B. Chittibabu (2015) are concluded also that the circuit training is useful for improving aerobic velocity on basketball players. The results of previous research are further reinforces that with the circuit method for 6 (six) weeks are compared with the control group 
significantly which had differences in cardiovascular activity (Raj Kumar, 2016). The three results of the study are physiologically related to the presence of factors stimulus training circuit. The consequences, in turn, can be has implications for physical enhancement.

It becomes empirically evident that circuit training can develop physical abilities. It is can be said that the method of circuit training as an efficient exercise tool to help lower fat body, increase insulin sensitivity and can increase fitnes $\mathrm{VO}_{2 \max }$ (Brett Killa and Chris Jordan, 2013). Research results from Vikesh Kumar also reinforce that circuit training can significantly improve the speed, of leg strength, arm strength and agility. The results of this research can developed the ability of the physical elements effectively, because there are several physical elements that can be increased at the same time. A similar study conducted by Hamid Arazi and Abbas Asadi show that circuit training is better to increase muscle endurance compared with condensed training multiple-set and the control group (Hamid Arazi and Abbas Asadi, 2012).

From theoretical and empirical study above, that circuit training methods are a method of training that can improve the physical elements. That's what makes the writer more interested in developing models of physical exercise with the circuit method. These elections saw the results of empirical studies, which claimed more circuit training method can enhance capabilities physical simultaneously. Physical improvement which involves some elements is then a reference to the author in developing the model of physical exercise keeper. Thus, in determining the use of training methods has a solid foundation.

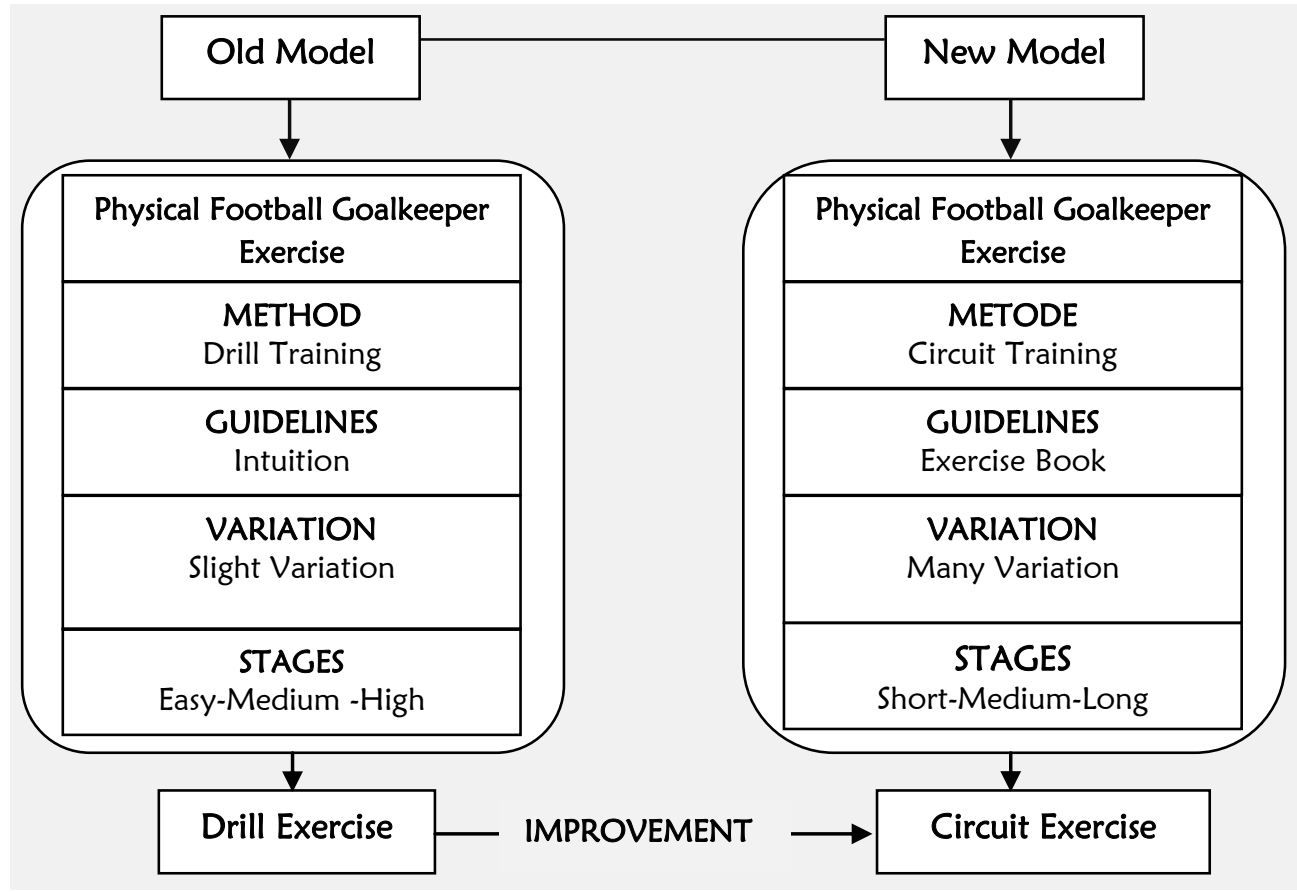

Picture 1. Comparison of Old and New models (Source: Authors) 
Pictured above is the result of a comparative analysis of models of exercise existing physical after seeing the results of field observations, with a model that will be developed in terms of theoretical studies and development plans. The existing model is more focused on method, training drill which tends to be a little variation. Another reason of the selection circuit training method, the author is convinced that physical exercise using more circuits can vary the elements, physical. It is the excess of the circuit method. That is why the use of the circuit method is based on the selection with theory a solid study. Thus the results of this development will be used as a guide in preparing the program of physical exercise goalkeeper of football.

\section{METHOD}

The research development of models of physical exercise goalie by methods circuit is using a model of research and development from Borg and Gall (1983) consisting of ten steps, namely:

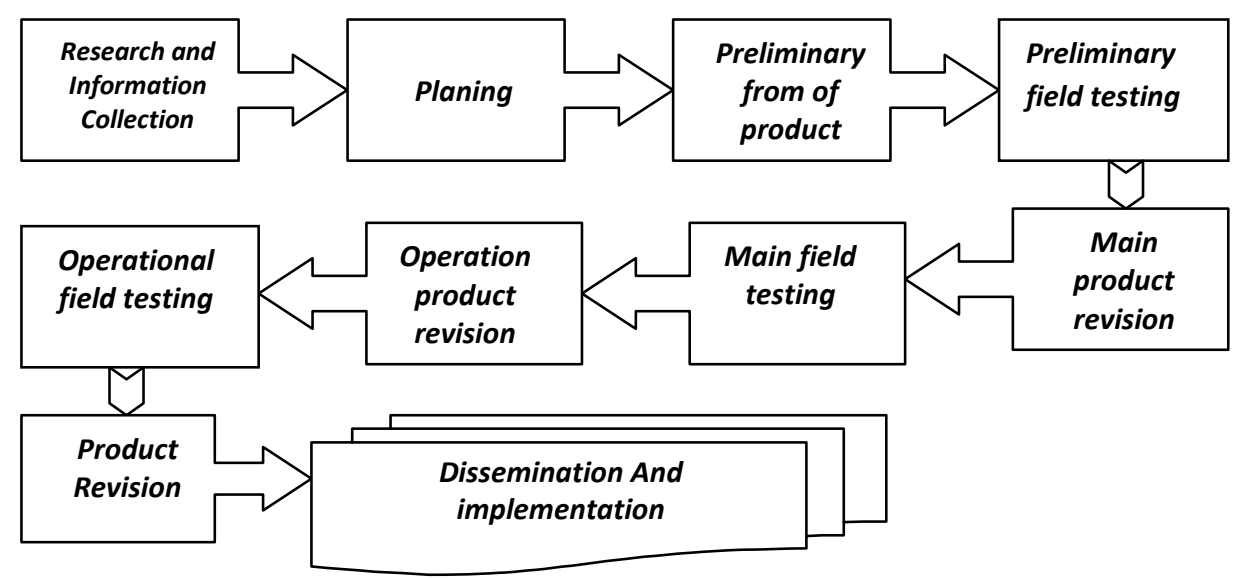

Picture 2 Stage Research and Development Source: WR Borg, DM Gall and Gall

Pictured above, a groove in research and development, Borg and Gall then describe in detail each of the steps in picture above and summarized by Ali Maksum (2011) and adapted to this research. So he found this explanation: (1). Collect information and the results of research: the study of literature, observation, and the disclosure of state of the art (2). Manage a research plan: as like to set goals, determine indicators of success, feasibility testing, the time and cost required. (3). Development of the first product: like making model of practice, guidelines and evaluation tools. (4). Preliminary field testing: Testing 1-3 basketball club uses 6-12 subjects. At the time it is going to collect data through observation, interviews, and questionnaires. The objective testing at this stage is to obtain feedback of qualitative on the feasibility of its initial products developed. 
(5). Revise the preliminary field testing: do the analysis and revision of results of preliminary field testing. (6). The main Friendlies (main field testing): testing 5-15 the club or football schools with 30-100 subjects to subjects. Perform pre and posttest and the use of an adequate control group by relying on quantitative data. Objective testing at this stage is to determine whether a product developed in accordance with the objectives are achieved. (7). Revision of the main trial results: doing analysis and revision of the test results. (8). Operational Tests (operational field testing): testing the 10-30 club or football schools involving 40-200 subject. At the same time also collected data through observation, interviews, and questionnaires. Objective testing at this stage is to determine whether the products developed are fully ready for use in clubs or football school even without the presence of the researcher. (9). Revision of the final product: do the analysis and revision of the results of test the field execution. (10). Dissemination and implementation: collated research reports and publications in scientific meetings or through journals, including the possibility to distribute the product in the form of a mass.

The ten steps above is the cycle in conducting research and development. At the same time as a reference in developing a model of physical exercise method circuit on goalkeeper of football. Another reason of the use of research and approach developments considered particularly to appropriate in developing a model of physical exercise, this is due to the development of a model based on the existing conditions in the field. So the selection of this approach is an option with a solid rationale.

\section{RESULTS}

\section{Needs Assesment}

A technique of data retrieval on a need analysis is based on interviews, observation and questionnaire-questionnaire. Interviews were conducted on soccer coaches with the aim to gather information, the observation is made with careful observation on the training process which has been ongoing, and questionnairequestionnaire is the result of distribution divided to athletes soccer goalkeeper.

Table.1 Need Assesment

\begin{tabular}{|c|c|}
\hline Method & Data Conclusion \\
\hline Interview & $\begin{array}{l}\text { - Interview with the coach stated that the lack of a guide } \\
\text { book for physical training goalkeepers } \\
\text { - } \quad \text { Difficult to find specific guidance physical training circuit } \\
\text { goalkeeper } \\
\text { - It takes a model of physical exercise circuit goalkeeper }\end{array}$ \\
\hline Observations & - It found that the exercise undertaken only lean on mere \\
\hline
\end{tabular}




\begin{tabular}{|l|l|}
\hline & $\begin{array}{l}\text { intuition nothing in mind at that time was what trained on } \\
\text { the spot. } \\
\text { Q }\end{array}$ \\
\hline Questionnaire & $\begin{array}{l}\text { Exercise is still similar to the specifications of the keeper. } \\
\text { from the athlete / goalkeeper shows the percentage as } \\
\text { much as } 64.96 \% \text { (Sixty-four point ninety-six percent) } \\
\text { From the distribution of a questionnaire-the questionnaire } \\
\text { can be interpreted that the development models of } \\
\text { physical exercise agree to be developed. }\end{array}$ \\
\hline
\end{tabular}

The method of collecting data as it existed in the table above, is a way to translate the need assessment (analysis of needs). The results of the analysis of the needs can be stated that the presence of physical exercise models goalkeeper with a circuit method, had to be developed in a special physical training the keeper.

\section{The Preliminary Draft Concept (Early Model)}

The purpose of the initial draft of concept to describe the design of the models developed. The concept of the preliminary draft can be seen in the table below

Table. 2 The concept of the Draft Preliminary

\begin{tabular}{|c|c|c|c|c|}
\hline Physical Needs & Principle of & \multicolumn{3}{|c|}{ The Exercise Methods of Fsik } \\
\hline \multirow{10}{*}{$\begin{array}{ll}\text { - } & \text { Reaction rates } \\
\text { - } & \text { Muscle } \\
\text { - } & \text { Strength } \\
& \text { Abdongth } \\
& \text { Muscle } \\
\text { - } & \text { Muscle } \\
& \text { Strength } \\
\text { - } & \text { Eximbs } \\
\text { - } & \text { Agilosive } \\
\text { - } & \text { Speed } \\
\text { - } & \text { Flexibility } \\
\text { - } & \text { Coordination }\end{array}$} & \multirow{9}{*}{$\begin{array}{ll}\text { - } & \text { Progressive } \\
\text { - } & \text { Specific } \\
\text { - } & \text { Development } \\
\text { - } & \text { Overload } \\
\text { - } & \text { Variation }\end{array}$} & $\begin{array}{l}\text { Short } \\
\text { Cicuit }\end{array}$ & $\begin{array}{l}\text { Medium } \\
\text { Circuit }\end{array}$ & $\begin{array}{c}\text { Long } \\
\text { Circuit }\end{array}$ \\
\hline & & $6 \operatorname{pos}(a)$ & $10 \operatorname{pos}(a)$ & $13 \operatorname{pos}(a)$ \\
\hline & & 6 pos (b) & $10 \operatorname{pos}(b)$ & 13 pos (b) \\
\hline & & $7 \operatorname{pos}(\mathrm{a})$ & $11 \operatorname{pos}(\mathrm{a})$ & $14 \operatorname{pos}(a)$ \\
\hline & & 7 pos (b) & 11 pos (b) & 14 pos (b) \\
\hline & & $8 \operatorname{pos}(a)$ & $12 \operatorname{pos}(a)$ & 15 pos (a) \\
\hline & & 8 pos (b) & $12 \operatorname{pos}(b)$ & 15 pos (b) \\
\hline & & 9 pos (a) & & \\
\hline & & 9 pos (b) & & \\
\hline & $\begin{array}{l}\text { Total method of } \\
\text { Exercise }\end{array}$ & $\begin{array}{c}8 \\
\text { Methods } \\
\text { of } \\
\text { Exercise }\end{array}$ & $\begin{array}{c}6 \\
\text { Methods } \\
\text { of } \\
\text { Exercise }\end{array}$ & $\begin{array}{c}6 \text { Methods } \\
\text { of } \\
\text { Exercise }\end{array}$ \\
\hline
\end{tabular}


Table above is the draft concept of the first (model) which has been made. The concept of the first draft was made as comfortable as possible by basing on the results of the needs analysis and theoretical studies. So, with results of draft concept of the first model there are 20 (twenty) of exercises with the circuit method. However, the concept of the initial draft in the table above have not yet signed the due diligence phase models. Stages of feasibility studies involving some competent experts in the art, namely expert coaching methodology and expert sports coaches of goalkeeper. The results of the feasibility test can be seen on the next point.

\section{Feasibility Model}

Testing the feasibility of a model involving five (5) experts who are competent in their field, that expert coaching methodology of sports 2 (two) people, and 3 (three) experts of goalkeeper. Interest test feasibility is intended to get the validation of the developed model. The results of the feasibility study are contained in the following table.

Table.3 Results of Feasibility Model

\begin{tabular}{|c|c|c|c|c|c|c|c|}
\hline \multicolumn{5}{|c|}{ Test Results Feasibility by Expert } & \multicolumn{3}{|c|}{ Training Methods } \\
\hline$* \mathbf{A 1}$ & $* \mathbf{A} 2$ & $* \mathbf{A 3}$ & $*$ A4 & $* \mathbf{A 5}$ & $\begin{array}{l}\text { Short } \\
\text { Circuit }\end{array}$ & $\begin{array}{l}\text { Medium } \\
\text { Circuit }\end{array}$ & $\begin{array}{c}\text { Long } \\
\text { Circuit }\end{array}$ \\
\hline Feasible & Feasible & Feasible & Feasible & Feasible & 6 pos (a) & $10 \operatorname{pos}(\mathrm{a})$ & 13 pos (a) \\
\hline Feasible & Feasible & Feasible & Feasible & Feasible & 6 pos (b) & $10 \operatorname{pos}(\mathrm{b})$ & 13 pos (b) \\
\hline Feasible & Feasible & Feasible & Feasible & Feasible & 7 pos (a) & 11 pos (a) & 14 pos (a) \\
\hline Feasible & Feasible & Feasible & Feasible & Feasible & 7 pos (b) & $11 \operatorname{pos}(b)$ & $14 \operatorname{pos}(\mathrm{b})$ \\
\hline Feasible & Feasible & Feasible & Feasible & Feasible & 8 pos (a) & $12 \operatorname{pos}(\mathrm{a})$ & 15 pos (a) \\
\hline Feasible & Feasible & Feasible & Feasible & Feasible & 8 pos (b) & $12 \operatorname{pos}(\mathrm{b})$ & 15 pos (b) \\
\hline Feasible & Feasible & Feasible & Feasible & Feasible & 9 pos (a) & & \\
\hline Feasible & Feasible & Feasible & Feasible & Feasible & 9 pos (b) & & \\
\hline \multicolumn{5}{|c|}{$\begin{array}{c}\text { Total Feasibility } \\
\text { Model }\end{array}$} & $\begin{array}{c}8 \\
\text { Method } \\
\text { Feasible }\end{array}$ & $\begin{array}{c}6 \\
\text { Methods } \\
\text { Feasible }\end{array}$ & \begin{tabular}{|c|}
6 \\
Methods \\
Feasible
\end{tabular} \\
\hline . & rs & & & & & & \\
\hline
\end{tabular}

Then, the revision results is made as a foothold in improving the model. After improving the exercise model with the instruction which has been submitted by the expert, the next step is testing a model that has been feasible to the athlete of soccer goalkeeper. The trial was conducted in small groups and large groups. 


\section{Effectiveness of Model}

This section also explains about the effectiveness test models. This test is performed with the involvement of athletes goalkeeper as many as 30 (thirty). The purpose of this test is to see how effective this type of training that has been generated. Data generated on the effectiveness of the test based on quantitative. Thus the experimental design is a must to use. The design used in this effectiveness test is using design randomized control group pretest-posttest design. Selection of design for the effectiveness test is to be a good choice, because the design is a purely experimental design with the characteristic of the group control taken at random from the 30 (thirty) goalkeepers involved. Then thirty-keepers is drawn at random, to be placed in the group of circuit training and training conventional group.

In this test does not use the way as like in small trials or large but to use quantitative data to see the results of the pretest-posttest and outcomes between groups. For this reason, it way is deemed an objective to assess the effectiveness of the model. Results of quantitative data as mentioned will be seen in the difference in the value of each training group. Thus it is necessary to test different (t-test) to see which is more effective between the physical training with circuit method of training conventional. Is it true model of exercise physical with the circuit method is more effective? Or is it a conventional training more effective. Therefore, to see the need to do things test different groups using independent t-test techniques. It means that it follows.

Table 4. Test Results Differences between Groups

\begin{tabular}{|l|c|c|c|c|c|c|}
\hline \multicolumn{1}{|c|}{ Training } & Variable & Group & Statistics & T-test & Sig & Status \\
\hline \multicolumn{2}{|c|}{} & N & Mean & \multicolumn{2}{|c|}{} \\
Model & Ability & 15 & 42.221 & 3.447 & 0.00 & $\begin{array}{c}\text { Differen } \\
\mathrm{t}\end{array}$ \\
\cline { 1 - 4 } Conventional & Physical & 15 & 33.167 & & & \\
\hline
\end{tabular}

From acquisition results of the above table of each group can be known mean value for groups of circuit models of 42221 and the value of the conventional group amounted to 33167 t-count value 3,447. Results thus it can be stated that the training method proved to be circuitry more can improve physical abilities soccer goalie when compared to conventional training. With this result emphasized that the circuit training model can stimulate physical abilities of keeper.

Based on the development of physical training goalie soccer with the circuit method was no doubt in the implementation of the field. Therefore 
goalkeeper coach if you want to develop physical abilities soccer goalkeeper, the method of this circuit is the right choice, because it has been tested and scientifically developed.

\section{DISCUSSION}

Having all that in-depth development of models of physical exercise with the circuit method is conducted. Studies carried out starting with their initial study needs analysis indicates that the presence of models physical exercise is very necessary in order to support the process of physical training on a soccer goalie. The study results are used as a foothold in doing the drafting of initial or early models. At the same time the drafting of initial or early models are also based on the results of theoretical studies. Then, getting the decision that the results of theoretical studies of physical exercise will be applied using the circuit method.

The use of this circuit method is not without reason, but it has been conducted in-depth theoretical studies that exercise physical circuit is considered effective in developing physical abilities goalkeeper, because all elements of the physical condition can be trained simultaneously. This is in line with the statement of Scholish that training circuits fitness training methods designed to develop general physical condition, as well as physical fitness and cardiovascular (Scholish, 1990). According to the theory of Wastcott Wayne stated that circuit training is a model exercise that involves a series of different exercises are carried out sequentially and continuously in one round or circuit (Wayne Wastcoot, 2003). The theory above can be said that training circuit can be called with a complex exercise because it involves many elements the physical components.

Besides, the availability of time within training that it tends to be very limited a distinct advantage for coaches and athletes, as with other forms of exercise circuit method can be integrated. This is why the circuit method should be developed as a way to resolve the issue. Underlying the results of needs analysis and the results of theoretical studies, they invented this early model draft, draft the initial model is validated by experts who are competent in their field before being tested athletes soccer goalkeeper. The early models which have been validated by experts are tested to the athletes of goalkeeper. Experiments carried out on a smaller scale and in a wider scale. From the results of both tests were subsequently carried out repairs of the revision carried out by experts.

After being conducted the small and widely test, the next step to determine the level of effectiveness of the model. For this purpose is necessary to test statistically. This test was deliberately conducted to determine the effective level of the developed model before the model is distributed to audiences. Models of physical exercise circuit on research and development is very specifically aimed at keeper. The Models of this exercise is designed in a way to help a goalkeeper in developing physical abilities.

In line with the model of this exercise also can help to design physical 
exercise of the keeper. The existence of this model also can make reference coach in giving menus physical exercise keeper. However, from the results of this exercise model development needs to consider the infrastructure used, because most of forms of training use tools as like cone, sphere, or use the stake as a medium to implement the circuit.

The result of models gets several supporting factors in applying physical exercise circuit. The supporting factor is the infrastructure used in the implementation of the model of a physical model circuit exercises are complete so that to make it easy to facilitate the application of models of physical exercise circuit. It becomes a separate record for each club and soccer school that if it needed to implement a circuit of physical exercise, it is necessary to consider of facilities and the use infrastructure.

In addition, in this study were also presented regarding the strengths and weaknesses of models produced. The strength of this model lies in increasing the physical ability to do together; this is a distinct advantage for the coach. The availability of time in the training process is sometimes classified short. Exercise Physical circuit becomes a solution to the availability of inadequate time. So, this circuit model of physical exercise is considered very effective in the developing components of the physical conditions football concurrently goalkeeper because in the process of training all of elements the physical can be trained on each of the posts that have been determined.

While the weak point of this model athlete tendency to haste in doing circuit training. This can be seen from the psychological side with the number of postal items either from a circuit workout short, medium and long circuit allows the athlete wants as soon as possible complete circuit workout. The role of the coach in this case it is important to sensitize athletes. Intensive communication with the athletes is something that needs to be done in order to realize the objectives of the exercise has been determined.

Overall, the model generated exercise has become a solution to the problems in the field. The model of this exercise was systematically conducted indepth, so it can be justified scientifically. This model is proved to improve physical ability keeper. It is also supported by the findings of research conducted by Syed Ali Syed Kamaruzaman, et.al (2015) shows that circuit training can improve muscle endurance. Results of another study also state that the circuit training had a significant impact on the strength of the abdominal muscles, arm and shoulder durability if it is compared to exrcise a speed, agility, and quickness. The results of Atul Meethal and Najeeb (2013) show that physical exercise can improve the circuit components of speed, agility, explosive leg power, pulse, blood pressure and aerobic capacity. The findings contained in the study and the results of the development model of physical exercise with the circuit method, can be one way for the coach if you want to develop abilities physical goalkeeper. So, the selection of circuit model of physical exercise is the right thing to do. 


\section{CONCLUSION}

The findings in the field by relying on the analysis of needs, in turn it has resulted in an answer to their products, including models of physical exercise in athletes circuit goalkeeper of soccer. Model physical exercise that has been made is as a way to overcome the gap between expectation and reality. Models exercise of physical in general is ready to be used in applying the physical exercise on the goalie. From various studies that have been conducted at the time of the study, physical exercise models specifically use the circuit.

Selection of the circuit method does not mean without basis, but it has been studied in depth the existing needs in the field. So it can be stated that the product model of physical exercise with using circuit is totally in accordance with the needs existing in the field. Thus, the resulting product is a efficient product, effective, and can be justified scientifically. The feasibility of the model which has been generated, is already the standards of feasibility, the feasibility level is got from the assessment of experts who are competent experts in their field.

Feasibility level is as a benchmark, whether the product of the reulted models is already as the criteria? Becuse in research and development, the feasibility of the product is a necessity. In a feasibility test whether the model also contains physical exercise with using the circuit method is in accordance with the principles of the exercise? From the expert assessment exercise model developed has met from the principles of exercise. In addition to the experts also considered in detail that started from the development of the model of physical exercise with short circuit method, medium and long circuit. This assessment is intended so that the items from the various models of physical exercise whether can be used properly? The results of the assessment of the experts shows that the model of the exercise really worthy to be applied and used. This is the more imposing that the model developed has fulfilled the standard to be applied in the field activities.

In addition, assessment on the effectiveness of the model becomes part of the groove Research and development. In review of the effectiveness, researchers are based on the quantitative data. Pre-test and post-test becomes a plurality of measuring the effectiveness of a model that has been produced. From the results that have been implemented in field indicates that there is a significant difference in the sole gatekeepers before and after treatment physical exercise using the circuit method. With the existence of the results, then physical exercise with circuit method can be stated that the model of this exercise is very effective used to improve physical on the sole gatekeepers of football.

\section{REFERENCES}

Atul Meethal and A .M.Najeeb. (2013). Effects Of Circuit Training On Different Surfaces On Selected Physical And Physiological Variables Of School Boys. International Journal of Physical Education, Fitness and Sports | Vol.2. No.4 | December. 
Borg. W. R and Gall, M. D. (1983). Educational Research : An Introduction. New York : Longman.

Brett Klika and Chris Jordan. (2013). High-Intensity Circuit Training Using Body Weight: Maximum Results With Minimal Investment. American College of Sports Medicine. Volume 17 No 3.

Hamid Arazi and Abbas Asadi. (2012). Multiple Sets resistance training: Effects of condensed versus circuit models on muscular strength, endurance and body composition. Journal of Human Sport \& Excercise. Volume 7 Issue 4

Maksum, Ali. (2012). Metodologi Penelitian dalam Olahraga (Surabaya: Unesa University Press.

Minakshi Pathak. (2015). Effects Of Badminton Specific Circuit Training On Selected Physiological Variables Of Badminton Players. International Journal of Sports Science, Fitness and Lesuire Industry. Volume 2 Issue I.

N. Akilan and B. Chittibabu. (2015). Effect Of Sports Specific Endurance Circuit Training On Maximal Aerobic Speed Of High School Male Basketball Players During Competitive Season. International Journal for Life Sciences and Educational Research. Vol.3 (1), 7-9, January.

Raj Kumar. (2016). Effect of 6 weeks circuit training on breath hold time of badminton players. International Journal of Advanced Education and Research. Volume 1; Issue 7; July.

Republika.co.id. (2014). Para Penjaga Gawang Bersinar di Piala Dunia 2014. Diakses 2 Juli

Scholich, M. (1990). Circuit Training For All Sports: Methodology Of Effective Fitness Training. (Sport Books Publisher: Toronto.

Syed Kamaruzaman Syed Ali, Malathyie A/P K. Arumugam, Zahra Ranjbar, Megat Ahmad KamaluddinMegatDaud, Rahmad Sukor Ab Sama. (2015). The effectiveness of circuit training in enhancing muscle endurance among standard five boys in a primary school. International Journal of Physical Education, Sports and Health; 2(1).

Vikesh Kumar. (2016). Effect Of Circuit Training Program On Selected Motor Abilities Among University Male. International Journal of Physical Education, Sports and Health; 3(4).

Wastcott, Wayne. (2003). Building Strength \& Stamina. (Human Kinetics. Massachusetts: Allyn and Bacon. Inc. 